\title{
Happy Mouth and Sad Eyes: Scanning Emotional Facial Expressions
}

\author{
Hedwig Eisenbarth \\ University of Regensburg
}

\author{
Georg W. Alpers \\ University of Mannheim and University of Würzburg
}

\begin{abstract}
There is evidence that specific regions of the face such as the eyes are particularly relevant for the decoding of emotional expressions, but it has not been examined whether scan paths of observers vary for facial expressions with different emotional content. In this study, eye-tracking was used to monitor scanning behavior of healthy participants while looking at different facial expressions. Locations of fixations and their durations were recorded, and a dominance ratio (i.e., eyes and mouth relative to the rest of the face) was calculated. Across all emotional expressions, initial fixations were most frequently directed to either the eyes or the mouth. Especially in sad facial expressions, participants more frequently issued the initial fixation to the eyes compared with all other expressions. In happy facial expressions, participants fixated the mouth region for a longer time across all trials. For fearful and neutral facial expressions, the dominance ratio indicated that both the eyes and mouth are equally important. However, in sad and angry facial expressions, the eyes received more attention than the mouth. These results confirm the relevance of the eyes and mouth in emotional decoding, but they also demonstrate that not all facial expressions with different emotional content are decoded equally. Our data suggest that people look at regions that are most characteristic for each emotion.
\end{abstract}

Keywords: emotion, facial expressions, eye-tracking, scan path
Facial expressions contain information relevant for social behavior. Thus, the processing of facial information is optimized (e.g., fast and automatic processing; Dimberg, Thunberg, \& Grunedal, 2002) and is carried out by specialized brain regions (e.g., fusiform face area; Kanwisher, Mcdermott, \& Chun, 1997). Although the exact nature of the specificity of facial processing is still being discussed (Hanson \& Halchenko, 2008), preferential processing of emotional facial expressions has been well documented (Alpers \& Gerdes, 2007; Pessoa, Kastner, \& Ungerleider, 2002; Vuilleumier, Armony, Driver, \& Dolan, 2001).

Most individuals are very effective in decoding emotional facial expressions; several studies using categorization tasks have demonstrated that happy faces are categorized nearly perfectly, whereas fearful facial expressions are classified slightly less perfectly (Calvo \& Lundqvist, 2008; Calvo \& Nummenmaa, 2009). The error rate increases as the number of emotional expressions presented in one experiment increases (Calvo \& Lundqvist, 2008; Eisenbarth, Alpers, Segrè, Calogero, \& Angrilli, 2008; Kreklewetz \& Roesch, 2005) and is, to some extent, dependent on culture

Hedwig Eisenbarth, Department of Forensic Psychiatry and Psychology, University of Regensburg, Regensburg, Germany; Georg W. Alpers, Chair of Clinical and Biological Psychology, School of Social Sciences, University of Mannheim, Mannheim, Germany and University of Würzburg, Würzburg, Germany.

The first author was funded by a scholarship from the KonradAdenauer-Foundation. We thank Kartin Blumenauer for helpful comments on the manuscript.

Correspondence concerning this article should be addressed to Dr. Hedwig Eisenbarth, University of Regensburg, Department of Forensic Psychiatry and Psychotherapy, Universitätsstrasse 84, D-93053 Regensburg, Germany. E-mail: hedwig.eisenbarth@medbo.de
(Jack, Blais, Scheepers, Schyns, \& Caldara, 2009). When misclassifications occur, they are often due to characteristic mix-ups. Fearful and surprised facial expressions are misclassified in approximately $10 \%$ of the trials and are classified as angry or disgusted facial expressions, and vice versa. An explanation for these mix-ups could be that those emotional expressions contain primary information about the emotional content in the eye region. Others could represent disgusted and fearful expressions that contain relevant information in regions like the nose or the forehead (see Jack et al., 2009).

The fact that complex information needs to be integrated in order to correctly classify emotional expressions becomes evident when the performance of healthy participants is compared with schizophrenic, autistic, psychopathic, or depressed patients; these patients are significantly less accurate in decoding. Schizophrenic patients score lower in decoding sad and angry expressions (Bediou et al., 2005; Streit, Wolwer, \& Gaebel, 1997), autistic patients mainly in sad facial expressions (Boraston, Blakemore, Chilvers, \& Skuse, 2007), psychopathic patients in negative facial expressions (Eisenbarth et al., 2008; Hastings, Tangney, \& Stuewig, 2008; Kosson, Suchy, Mayer, \& Libby, 2002), and depressed patients in happy facial expressions (Joormann \& Gotlib, 2006). This impairment may result from the fact that these patients process stimuli differently compared to healthy controls. A crucial part of such a processing difference may begin at the behavioral level, that is, in different ways of examining faces. Indeed, whereas healthy individuals mainly examine the eyes and mouth when looking at facial expressions (Henderson, Williams, \& Falk, 2005; Yarbus, 1967), scan paths reveal that the patient groups listed above often show a deviant scan path with no preference for a specific facial area (Dalton et al., 2005; Hernandez et al., 2009; Loughland, Williams, \& Gordon, 2002; Streit et al., 1997).

Similar to these patient groups, certain circumscribed brain lesions have also been shown to be accompanied by impaired 
decoding accuracy. For example, a patient who suffered circumscribed amygdala damage, S.M., evidenced a specific deficit in decoding fear expressions (Adolphs et al., 2005). This case is particularly interesting because recent data show that the patient's impairment when judging emotions is related to an inability to make normal use of information obtained through the eye region of a face. Interestingly, this defect was traced back to a lack of spontaneous fixation of the eyes during free viewing of faces (Adolphs et al., 2005).

One method to examine attentional allocation is by monitoring eye gaze. Eye movements and spatial attention are inextricably linked (Engbert \& Kliegl, 2003; Jonides, 1981; Klein, 2004; Rizzolatti, Riggio, Dascola, \& Umilta, 1987; Smith, Rorden, \& Jackson, 2004), and people usually do not engage in an effortful dissociation when they are free to move their eyes (Findlay \& Gilchrist, 1998; Rayner, 1998). Importantly, even the first saccade can be guided by global information about a scene background or setting glimpsed at picture onset (Rayner \& Pollatsek, 1992; Rousselet, Joubert, \& Fabre-Thorpe, 2005). Viewing emotional pictures clearly results in different scan paths compared with neutral scenes (Alpers, 2008; Nummenmaa, Hyona, \& Calvo, 2006). A direct link between scan paths and emotional response has been documented by the emotionality experienced when individuals view chimeric facial expressions (Butler et al., 2005) and in patient groups, such as socially anxious individuals, in which the gaze directed to the eyes is related to higher physiological responding (Wieser, Pauli, Alpers, \& Mühlberger, 2009). The relevance of facial scan paths to social interaction has been highlighted by the observation that stigmatization of people with facial deformities corresponds with deviant scan paths compared with normal faces (Meyer-Marcotty, Alpers, Gerdes, \& Stellzig-Eisenhauer, 2010; Meyer-Marcotty, Gerdes, Stellzig-Eisenhauer, \& Alpers, in press).

Because most of the studies reporting scan path data refer to stimuli with neutral facial expressions, the aim of the present study was to examine scan paths of healthy individuals while they examined different facial expressions representing basic emotions and to investigate whether there are emotion-specific scan paths.

\section{Method}

\section{Participants}

Thirty-six psychology students (20 female, 16 male) were recruited at the University of Würzburg and received course credit for their participation. Their mean age was 22.11 years $(S D=$
3.81; range: 18 to 33), all were native German speakers, 32 of the participants were right-handers, 3 were left-handers, and 1 was ambidextrous. Fifteen participants had corrected nearsightedness (eight using contact lenses), and 21 had normal vision. The mean score of the Chimeric Faces Test (CFT; Levy, Heller, Banich, \& Burton, 1983) was $M=-0.21$ ( $S D=0.40$; range: -1.00 to 0.81$)$, which corresponds to the normal left-bias for emotion detection literature. The mean score of the Social Phobia and Anxiety Inventory (Turner, Beidel, Dancu, \& Stanley, 1989) was $M=2.07$ $(S D=0.72$; range: 0.47 to 3.77$)$, and the mean score of the Trait Anxiety Inventory (Spielberger, Gorsuch, Lushene, Vagg, \& Jacobs, 1983) was $M=38.43$ ( $S D=9.16$; range: 22 to 65$)$, both within the normal range. All participants gave written informed consent.

\section{Material and Apparatus}

Stimuli were chosen from the Karolinska Directed Emotional Faces set (Lundqvist, Flykt, \& Ohman, 1998), which has proven to reliably evoke specific emotions (Goeleven, De Raedt, Leyman, \& Verschuere, 2008) with relatively naturalistic emotional expressions (Adolph \& Alpers, 2010). Eight female and eight male actors, each depicting afraid, angry, happy, neutral and sad emotional expressions, were included in a rating task (see Figure 1). Trials consisted of a picture presentation for $2,500 \mathrm{~ms}$, followed by a valence rating display ("How positive or negative is this picture for you?") (scale ranging from -4 to +4 ) and a subsequent arousal rating display ("How emotional arousing is this picture for you?") (scale ranging from 1 to 9). Ratings were done by key presses on a prepared keyboard.

Eye movements were recorded by a monocular video-based, high-speed tracking system with $1250 \mathrm{~Hz}$, (iView X Hi-Speed, SMI, Berlin, Germany). Participants were seated in front of the computer screen, the chin was placed on a chin rest to view the computer screen through the mirror-glass, which was used to reflect the eye, and the infrared light point tracked the eye movements with the camera placed above (for more details, see: Alpers, 2008). Location, time, and duration of all fixations were analyzed. Eight different areas of interest (AOI) were defined: forehead, left eye, right eye, left cheek, nose, right cheek, mouth, chin, and any other parts of the head. Fixations were defined as a gaze that remained in a diameter of 25 pixels for at least $100 \mathrm{~ms}$.

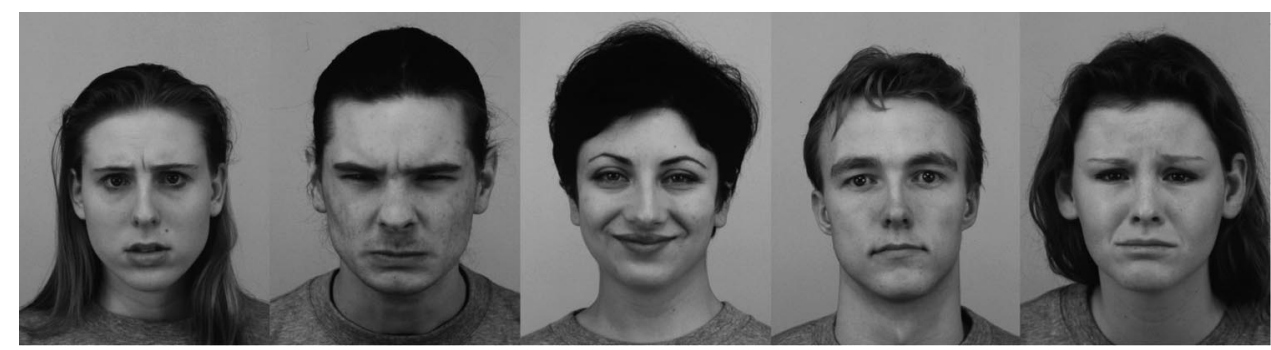

Figure 1. Exemplary stimuli (afraid, angry, happy, neutral, sad) chosen from the KDEF picture set (Lundqvist et al., 1998). 


\section{Data Analysis}

Fixation durations for each AOI, for the entire presentation period, were included in analyses of variance with a factor area of interest (eight steps) and a factor emotion (five steps). Fixation frequencies for each area of interest, for the first and second fixation as well as for the entire duration of a presentation, were included in Friedman tests for ranks, as the data consist of ordinal variables. Subsequently, in order to test the main hypotheses AOIs were reduced to the main areas: the eye and mouth regions.

A direct comparison between the frequencies of fixations issued to the AOIs was not possible because they were not statistically independent. Ratios were therefore computed for both frequencies of second fixations and fixation durations by dividing the difference of fixation numbers and the fixation durations of the eyes and mouth by the sum of the fixation numbers and fixation durations of the eyes and mouth, respectively. Thus, a positive ratio indicates that there were more or longer fixations on the eyes, whereas negative ratios depicted more or longer fixations on the mouth. Bonferroni-corrected follow-up tests were conducted for significant main effects and interactions.

\section{Results}

\section{Mean Fixation Duration}

Mean fixation duration differed between AOI, $F(1,35)=$ $115.58 ; p<.001 ; \eta^{2}=.77$. There was no main effect for emotion, but for an interaction between emotion and AOI, $F(4,140)=3.26$; $p=.01 ; \eta^{2}=.09$. Follow-up analyses for the main effects of AOI revealed significantly longer durations of the left and right eye compared to the forehead [left: $T(35)=-5.66 ; p<.001$; right: $T(35)=-5.66 ; p<.001]$, of the left and right eye compared to the left cheek [left: $T(32)=5.19 ; p<.001$; right: $T(32)=5.91$; $p<.001$ ], and of the left and right eye compared to the nose [left: $T(35)=3.74 ; p=.001$; right: $T(35)=4.52 ; p<.001]$. For the mouth region, fixations were significantly longer compared to fixations on the forehead, $T(35)=-3.91 ; p<.001$, the left cheek, $T(35)=-5.22 ; p<.001$, and the nose, $T(35)=-4.09$; $p<.001$. However, there were no significant differences between fixations on the mouth or the right cheek (see Table 1).

Follow-up tests for the interaction between AOI and emotion revealed significant effects for all five emotional expressions in the mean fixation duration of the AOI: fearful, $F(7,77)=6.68 ; p<$
$.001 ; \eta^{2}=.38$, angry, $F(7,77)=3.80 ; p=.001 ; \eta^{2}=.26$, happy, $F(7,84)=2.41 ; p=.03 ; \eta^{2}=.17$, neutral, $F(7,70)=5.95 ; p<$ $.001 ; \eta^{2}=.37$, and sad, $F(7,91)=3.01 ; p=.01 ; \eta^{2}=.19$. Separate tests, testing the factor emotion for each AOI, showed significant results only for the right eye, $F(4,132)=3.15 ; p=.02$; $\eta^{2}=.09$, but for no other facial region (see Table 1 ).

\section{Mean Number of Fixations}

The mean number of fixations revealed a main effect for AOI, $F(1,35)=111.58 ; p<.001 ; \eta^{2}=.76($ see Figure 2$)$ and a trend for an interaction between AOI and emotion, $F(4,140)=2.09$; $p=.09 ; \eta^{2}=.06$. There was no main effect for emotion. Follow-up tests concerning the main effect for AOI by testing differences in the numbers of fixations were significant for comparisons of the left or right eye and the forehead [left: $T(35)=$ $-5.30 ; p<.001$; right: $T(35)=-5.15 ; p<.001]$, as well as the left cheek [left: $T(35)=5.87 ; p<.001$; right: $T(35)=5.82 ; p<$ .001 ], and the nose [left: $T(35)=4.12 ; p<.001$; right: $T(35)=$ $4.35 ; p<.001]$. There were also more fixations on the mouth compared to the left cheek, $T(32)=-4.64 ; p<.001$ or the nose, $T(35)=-3.69 ; p<.001$.

\section{Location of First and Second Fixations}

When participants first looked at the screen at the beginning of each trial, the very first fixation was most frequently issued to an area outside of the face, including hair, ears, and throat, $\chi^{2}(7$, 36) $=112.76 ; p<.001$. The locations of the first and second fixations did not differ between emotional expressions. However, the second fixation was more often issued to one of the facial AOIs. There was a main effect for AOI, $\chi^{2}(7,36)=48.18 ; p<$ .001 ; the frequency at which they were looked at was the following: left eye, right eye, mouth, nose, hair, forehead, left cheek, and right cheek. There was no significant difference in the ranking between the eyes and mouth, but significantly fewer second fixations were issued to all of the other regions compared with the eyes (e.g., left eye-forehead: $Z=-3.27 ; p<.001$; right eye-forehead: $Z=-2.77 ; p=.01)$.

The ranking order for the AOI of each emotion yields differences between expressions. In $\operatorname{sad}\left[\chi^{2}(7,36)=43.57 ; p<.001\right]$ and angry facial expressions $\left[\chi^{2}(7,36)=26.15 ; p<.001\right]$, the left and right eyes were significantly more often the targets of the second fixation compared with all other regions. In fearful $\left[\chi^{2}(7\right.$,

Table 1

Mean Fixation Durations ( \pm Standard Deviations) for Each Category of Emotional Facial Expressions and Each Area of Interest

\begin{tabular}{llllll}
\hline & \multicolumn{5}{c}{ Facial expression } \\
\cline { 2 - 6 } \multicolumn{1}{c}{ AOI } & \multicolumn{1}{c}{ Fearful } & Angry & Happy & Neutral & Sad \\
\hline Forehead & $194.75 \pm 79.55$ & $188.70 \pm 45.96$ & $239.25 \pm 150.97$ & $166.83 \pm 16.73$ & $255.40 \pm 54.59$ \\
Left eye & $524.81 \pm 51.78$ & $578.03 \pm 177.20$ & $620.56 \pm 341.45$ & $541.79 \pm 66.41$ & $635.50 \pm 141.28$ \\
Right eye & $787.24 \pm 189.07$ & $757.50 \pm 120.92$ & $615.18 \pm 8.36$ & $722.26 \pm 113.98$ & $844.44 \pm 137.16$ \\
Left cheek & $336.75 \pm 3.18$ & $272.33 \pm 199.88$ & $257.25 \pm 90.86$ & $305.60 \pm 10.75$ & $477.50 \pm 79.90$ \\
Nose & $348.10 \pm 77.64$ & $335.00 \pm 108.19$ & $281.83 \pm 27.34$ & $235.82 \pm 82.98$ & $320.63 \pm 196.05$ \\
Right cheek & $462.56 \pm 33.16$ & $383.56 \pm 40.70$ & $431.98 \pm 130.78$ & $425.21 \pm 151.93$ & $511.72 \pm 158.78$ \\
Mouth & $427.25 \pm 72.48$ & $327.64 \pm 30.20$ & $416.32 \pm 93.79$ & $396.04 \pm 63.98$ & $359.29 \pm 77.02$ \\
Hair & $354.28 \pm 125.90$ & $434.61 \pm 203.73$ & $408.51 \pm 60.74$ & $394.12 \pm 52.66$ & $395.91 \pm 192.03$ \\
\hline
\end{tabular}




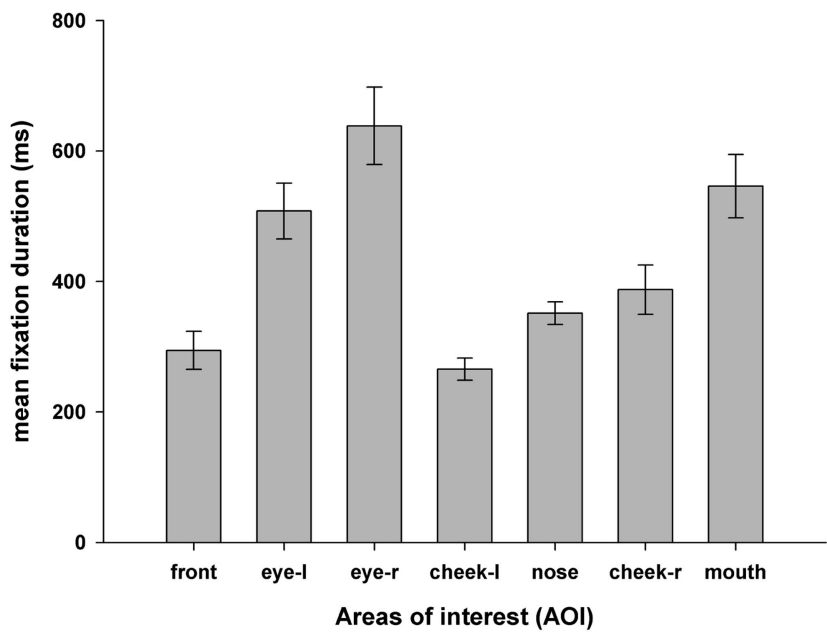

Figure 2. Mean fixation duration (ms) for each area of interest and standard error of the mean for all emotional facial expressions (front $=$ forehead; eye-1 = left eye; eye-r = right eye; cheek-1 = left cheek; cheek-r = right cheek).

36) $=40.56 ; p<.001]$ and neutral $\left[\chi^{2}(7,36)=41.29 ; p<.001\right]$ facial expressions, the mouth region was the target of the second fixation. In addition to these findings, in happy facial expressions, the mouth and hair regions were fixated upon significantly more often compared with the cheeks, nose, and forehead, $\chi^{2}(7,36)=$ $44.31 ; p<.001$.

\section{Ratios for Fixation Duration and Frequencies}

The ratios of total fixation duration and fixation frequencies were calculated in order to directly compare the emotion-relevant differences in the main facial regions. For the ratio of total fixation duration, there was a significant main effect of emotion, $F(4$, $140)=3.87 ; p=.01 ; \eta^{2}=.10$. Follow-up tests revealed a higher ratio for fearful facial expressions compared to happy ones, $T$ $(35)=3.72 ; p=.001$, and a higher ratio for sad facial expressions compared to happy ones, $T(35)=-2.83 ; p=.01$. Higher ratios represent longer fixation times of the eye regions compared to the mouth region (see Figure 3 ). There was a significant emotion effect on the frequencies of second fixations, $\chi^{2}(4,36)=10.54$; $p=.03$, which can be explained by a significant difference between sad facial expressions and fearful $(Z=-2.08 ; p=.04)$, angry $(Z=-2.39 ; p=.02)$, and happy $(Z=-2.11 ; p=.04)$ facial expressions.

\section{Discussion}

The way we look at faces has spurred scientific interest for many years (Henderson et al., 2005; Yarbus, 1967). Additionally, deviations in the scan paths of specific patient groups when looking at emotional facial expressions have been linked to their specific deficits in reading emotional faces (Dalton et al., 2005; Hernandez et al., 2009). The aim of this study was to examine differences in scan paths when healthy individuals look at different emotional facial expressions. Across all trials, primary target regions were the eye and mouth regions, including the photographed person's right cheek. For the duration of all fixations during picture presentation, participants fixated on the mouth region longer in happy facial expressions compared with sad and fearful facial expressions. The number of fixations showed that the eye region is most frequently fixated upon in all emotional expressions, but in fearful, happy, and neutral facial expressions, the mouth and right cheek regions are also fixated upon as frequently as the eye region. In sad and angry facial expressions, the eye region is most frequently fixated upon. Concerning the first fixation on the face (= second fixation in general) in sad facial expressions, participants more often looked at the eyes in comparison with all other emotional expressions.

The ratios that we computed for fixation durations on the eyes in relation to the mouth region underline these findings in a more direct way. The relative duration of fixations in the eye region is dominant, but less dominant for happy facial expressions compared to fearful, neutral, and sad expressions. In terms of the numbers of first fixations, the eye region is less dominant compared to the mouth region in angry facial expressions compared to sad expressions.

These results are in accordance with previous studies comparing healthy participants and patients. In healthy participants, the gaze is directed more often to the eye and mouth regions compared with other regions of a facial expression (see also Spezio, Adolphs, Hurley, \& Piven, 2007). According to these authors, the eye region is fixated upon more often and for longer durations compared with the other facial regions, independent of emotional category. This finding is plausible because important cues for emotional information can be found in this region (Ekman \& Friesen, 1969).

Striking evidence for the informational content of the eyes comes from studies that ask participants to define someone's emotional state just from seeing the eye region in scan paths when different emotional expressions are examined. In sad facial expressions, the first fixation on the face is to the eyes; in happy facial expressions, the mouth region is fixated upon longer compared with the other emotional expressions. Thus, if participants want to

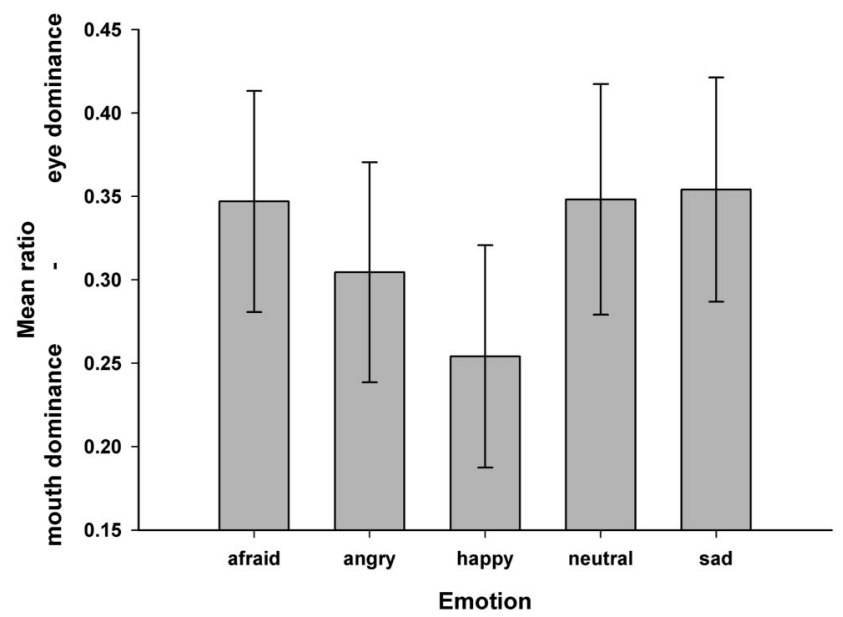

Figure 3. Mean ratio of fixation duration (duration for eyes - duration for mouth/duration for eyes + duration for mouth) for each emotional category and standard error of the mean; larger ratios indicate longer durations for the eyes compared to the mouth. 
decide for valence and arousal of a facial expression, eye gaze is directed to those regions where important emotion-specific information can be found: the smiling mouth or the sad eyes. This observation supports the idea that it is highly important for social interaction to know the emotional state of another person, and it facilitates good emotion discrimination capacities that have been found in different experimental paradigms (Calvo \& Lundqvist, 2008; Calvo \& Nummenmaa, 2009). Although these results seem trivial, to our knowledge, there is no study investigating the scan path in facial expressions presenting different emotional states.

However, it is not expected that the right cheek is as often fixated upon and for as long a duration as the mouth region. One explanation could be that, although insignificant, the number and durations of fixations in our data follow a right bias, which could explain higher exploration of the right cheek. This finding would not be in line with previous results, where predominantly left biases have been found in face perception (Gilbert \& Bakan, 1973; Levy et al., 1983). The dominance of the right cheek could also be due to the proximity of the mouth region to both cheek regions and, therefore, might be an artifact of choosing stable areas of interest for analysis. This would still suggest a right bias for the lower facial region across all emotional categories, although participants showed predominantly the more common left-bias in the CFT. A hypothesis concerning the right bias could be that the left-bias is more related to the initial processing of faces and especially related to reaction times, whereas long periods of exploration time are less related to this bias. In our data, the left eye descriptively but insignificantly was more often the target of first relevant fixation, which would support the hypothesis. A right-bias in face perception has been found to be present for positive facial expressions (Workman, Peters, \& Taylor, 2000) and for face recognition (Laeng \& Rouw, 2001). We did not find any differences between positive and negative facial expressions in this tendency of a right bias, but the task of evaluating the pictures for valence and arousal could relate to left hemisphere dominance.

A recent study with healthy individuals found that known faces can be recognized with only one fixation (Hsiao \& Cottrell, 2008). This first fixation is located above chance at the nose region of the picture. As soon as the authors gave participants time for more fixations to explore the face, recognition performance was further enhanced. Second fixations also have been found to be located above chance at the nose region, whereas the third fixation was directed toward the eye region. The authors conclude that these findings point to a holistic perception of facial expressions. Although in this study no emotional facial expressions were used, again the eye region appears to have a strong impact on face recognition. However, this study raises the question if face perception is still holistic when participants are asked to decide which emotion is being displayed and not if they know the person. Thus, future studies should address this query by answering the question of how many fixations are needed to decide the emotional content of a facial expression.

The present study confirms that the eyes are particularly important to read emotional expressions. Yet, some limitations must be considered. First, we did not experimentally control for the starting point of the scan path in each trial. Often, experiments accomplish such control with the help of a fixation cross before picture presentation. Instead, we decided to examine scan paths under more naturalistic viewing conditions and without such an experi- mental constraint. Second, subsequent studies should not only include arousal and valence ratings but also classifications of the expressions in order to include classification performance. In addition, presentation time should be varied, starting with very brief presentations to determine how many fixations are needed to correctly identify the emotional content of a facial expression. This addition could add relevant evidence to aid in the understanding of the processing of social and emotional contents.

In sum, our study supports the importance of the eye and mouth regions to facial perception. Moreover, it extends our knowledge in showing that scan paths of healthy observers differ for different emotional facial expressions. This specificity of gaze pattern may be due to specific emotional cues provided by specific regions of the face.

\section{References}

Adolph, D., \& Alpers, G. W. (2010). Differences in valence and arousal: A comparison of two sets of emotional facial expressions. American Journal of Psychology, 123, 209-219.

Adolphs, R., Gosselin, F., Buchanan, T. W., Tranel, D., Schyns, P., \& Damasio, A. R. (2005). A mechanism for impaired fear recognition after amygdala damage. Nature, 433(7021), 68-72. doi: 10.1038/ nature 03086

Alpers, G. W. (2008). Eye-catching: Right hemisphere attentional bias for emotional pictures. Laterality: Asymetries of Body, Brain and Cognition, 13, 158-178. doi: 10.1080/13576500701779247

Alpers, G. W., \& Gerdes, A. B. M. (2007). Here's looking at you: Emotional faces predominate in binocular rivalry. Emotion, 7(3), 495506. doi: 10.1037/1528-3542.7.3.495

Bediou, B., Franck, N., Saoud, M., Baudouin, J.-Y., Tiberghien, G., Dalery, J., et al. (2005). Effects of emotion and identity on facial affect processing in schizophrenia. Psychiatry Research, 133(2-3), 149-157. doi: 10.1016/j.psychres.2004.08.008

Boraston, Z., Blakemore, S.-J., Chilvers, R., \& Skuse, D. (2007). Impaired sadness recognition is linked to social interaction deficit in autism. Neuropsychologia, 45(7), 1501-1510. doi: 10.1016/j.neuropsychologia .2006.11.010

Butler, S., Gilchrist, I. D., Burt, D. M., Perrett, D. I., Jones, E., \& Harvey, M. (2005). Are the perceptual biases found in chimeric face processing reflected in eye-movement patterns? Neuropsychologia, 43(1), 52-59. doi: 10.1016/j.neuropsychologia.2004.06.005

Calvo, M. G., \& Lundqvist, D. (2008). Facial expressions of emotion (KDEF): Identification under different display-duration conditions. $B e-$ havior Research Methods, 40, 109-115. doi: 10.3758/BRM.40.1.109

Calvo, M. G., \& Nummenmaa, L. (2009). Eye-movement assessment of the time course in facial expression recognition: Neurophysiological implications. Cognitive, Affective, \& Behavioral Neuroscience, 9(4), 398 411. doi: $10.3758 /$ cabn.9.4.398

Dalton, K. M., Nacewicz, B. M., Johnstone, T., Schaefer, H. S., Gernsbacher, M. A., Goldsmith, H. H., et al. (2005). Gaze fixation and the neural circuitry of face processing in autism. Nature Neuroscience, 8(4), 519-526. doi: 10.1038/nn1421

Dimberg, U., Thunberg, M., \& Grunedal, S. (2002). Facial reactions to emotional stimuli: Automatically controlled emotional responses. Cognition \& Emotion, 16(4), 449-471. doi: 10.1080/02699930143000356

Eisenbarth, H., Alpers, G. W., Segrè, D., Calogero, A., \& Angrilli, A. (2008). Categorization and evaluation of emotional faces in psychopathic women. Psychiatry Research, 159(1-2), 189-195. doi: 10.1016/ j.psychres.2007.09.001

Ekman, P., \& Friesen, W. V. (1969). The repertoire of nonverbal behavior: Categories, origins, usage, and coding. Semiotica, 1(1), 49-98.

Engbert, R., \& Kliegl, R. (2003). Microsaccades uncover the orientation of 
covert attention. Vision Research, 43(9), 1035-1045. doi: 10.1016/ S0042-6989(03)00084-1

Findlay, J. M., \& Gilchrist, I. D. (1998). Eye guidance and visual search. In G. Underwood (Ed.), Eye guidance in reading and scene perception, (pp. 297-314). Amsterdam: Elsevier.

Gilbert, C., \& Bakan, P. (1973). Visual asymmetry in perception of faces. Neuropsychologia, 11(3), 355-362. doi: 10.1016/0028-3932(73) 90049-3

Goeleven, E., De Raedt, R., Leyman, L., \& Verschuere, B. (2008). The Karolinska Directed Emotional Faces: A validation study. Cognition \& Emotion, 22(6), 1094-1118. doi: 10.1080/02699930701626582

Hanson, S. J., \& Halchenko, Y. O. (2008). Brain reading using full brain support vector machines for object recognition: There is no "face" identification area. Neural Computation, 20(2), 486-503. doi: 10.1162/ neco.2007.09-06-340

Hastings, M. E., Tangney, J. P., \& Stuewig, J. (2008). Psychopathy and identification of facial expressions of emotion. Personality and Individual Differences, 44(7), 1474-1483. doi: 10.1016/j.paid.2008.01.004

Henderson, J. M., Williams, C. C., \& Falk, R. J. (2005). Eye movements are functional during face learning. Memory \& Cognition, 33(1), $98-$ 106.

Hernandez, N., Metzger, A., Magnè, R., Bonnet-Brilhault, F., Roux, S., Barthelemy, C., et al. (2009). Exploration of core features of a human face by healthy and autistic adults analyzed by visual scanning. Neuropsychologia, 47(4), 1004-1012. doi: 10.1016/j.neuropsychologia .2008 .10 .023

Hsiao, J. H.-W., \& Cottrell, G. W. (2008). Two fixations suffice in face recognition. Psychological Science, 19(10), 998-1006. doi: 10.1111/ j.1467-9280.2008.02191.x

Jack, R. E., Blais, C., Scheepers, C., Schyns, P. G., \& Caldara, R. (2009). Cultural confusions show that facial expressions are not universal. Current Biology, 19(18), 1543-1548. doi: 10.1016/j.cub.2009.07.051

Jonides, J. (1981). Voluntary vs. automatic control over the mind's eye's movement. In J. B. Long \& A. D. Baddeley (Eds.), Attention and performance IX (pp. 187-203). Hillsdale, NJ: Erlbaum.

Joormann, J., \& Gotlib, I. H. (2006). Is this happiness I see? Biases in the identification of emotional facial expressions in depression and social phobia. Journal of Abnormal Psychology, 115(4), 705-714. doi: 10.1037/0021-843X.115.4.705

Kanwisher, N., McDermott, J., \& Chun, M. M. (1997). The fusiform face area: A module in human extrastriate cortex specialized for face perception. Journal of Neuroscience, 17(11), 4302-4311.

Klein, R. M. (2004). On the Control of Visual Orienting. In M. I. Posner (Ed.), Cognitive neuroscience of attention (pp. 29-44). New York: Guilford Press.

Kosson, D. S., Suchy, Y., Mayer, A. R., \& Libby, J. (2002). Facial affect recognition in criminal psychopaths. Emotion, 2(4), 398-411. doi: 10.1037//1528-3542.2.4.398

Kreklewetz, K., \& Roesch, R. (2005, July). Facial affect recognition in psychopathic offenders. Paper presented at the Conference of the Society for the Scientific Study of Psychopathy, Vancouver.

Laeng, B., \& Rouw, R. (2001). Canonical views of faces and the cerebral hemispheres. Laterality, 6(3), 193-224. doi: 10.1080/713754410

Levy, J., Heller, W., Banich, M. T., \& Burton, L. A. (1983). Asymmetry of perception in free viewing of chimeric faces. Brain and Cognition, 2(4), 404-419. doi: 10.1016/0278-2626(83)90021-0

Loughland, C. M., Williams, L. M., \& Gordon, E. (2002). Schizophrenia and affective disorder show different visual scanning behavior for faces: A trait versus state-based distinction? Biological Psychiatry, 52(4), 338-348. doi: 10.1016/S0006-3223(02)01356-2

Lundqvist, D., Flykt, A., \& Ohman, A. (1998). Karolinska directed emo- tional faces $(K D E F)$. Stockholm, Sweden: Department of Neurosciences.

Meyer-Marcotty, P., Alpers, G. W., Gerdes, A. B., \& Stellzig-Eisenhauer, A. (2010). Impact of facial asymmetry in visual perception: A 3-dimensional data analysis. American Journal of Orthodontics \& Dentofacial Orthopedics, 137(2), 161-169. doi: 10.1016/j .ajodo.2008.11.023

Meyer-Marcotty, P., Gerdes, A. B. M., Stellzig-Eisenhauer, A., \& Alpers, G. W. (in press). How orthognathic patients are perceived by others: An eye-tracking study. World Journal of Orthodontics.

Nummenmaa, L., Hyona, J., \& Calvo, M. G. (2006). Eye movement assessment of selective attentional capture by emotional pictures. Emotion, 6(2), 257-268. doi: 10.1037/1528-3542.6.2.257

Pessoa, L., Kastner, S., \& Ungerleider, L. G. (2002). Attentional control of the processing of neutral and emotional stimuli. Cognitive Brain Research, 15(1), 31-45. doi: 10.1016/S0926-6410(02)00214-8

Rayner, K. (1998). Eye movements in reading and information processing: 20 years of research. Psychological Bulletin, 124(3), 372-422.

Rayner, K., \& Pollatsek, A. (1992). Eye movements and scene perception. Canadian Journal of Psychology, 46(3), 342-376.

Rizzolatti, G., Riggio, L., Dascola, I., \& Umilta, C. (1987). Reorienting attention across the horizontal and vertical meridians: Evidence in favor of a premotor theory of attention. Neuropsychologia, 25(1), 31-40. doi: 10.1016/0028-3932(87)90041-8

Rousselet, G., Joubert, O., \& Fabre-Thorpe, M. (2005). How long to get to the "gist" of real-world natural scenes? Visual Cognition, 12(6), 852877. doi: $10.1080 / 13506280444000553$

Smith, D. T., Rorden, C., \& Jackson, S. R. (2004). Exogenous orienting of attention depends upon the ability to execute eye movements. Current Biology, 14(9), 792-795. doi: 10.1016/j.cub.2004.04.035

Spezio, M. L., Adolphs, R., Hurley, R. S. E., \& Piven, J. (2007). Analysis of face gaze in autism using "Bubbles". Neuropsychologia, 45(1), 144 151. doi: 10.1016/j.neuropsychologia.2006.04.027

Spielberger, C. D., Gorsuch, R. L., Lushene, R., Vagg, P. R., \& Jacobs, G. A. (1983). Manual for the State-Trait Anxiety Inventory, Palo Alto: Consulting Psychologists Press.

Streit, M., Wolwer, W., \& Gaebel, W. (1997). Facial-affect recognition and visual scanning behavior in the course of schizophrenia. Schizophrenia Research, 24(3), 311-317. doi: 10.1016/S0920-9964(96)00126-0

Turner, S. M., Beidel, D. C., Dancu, C. V., \& Stanley, M. A. (1989). An empirically derived inventory to measure social fears and anxiety: The Social Phobia and Anxiety Inventory. Psychological Assessment, 1(1), 35-40. doi: 10.1037//1040-3590.1.1.35

Vuilleumier, P., Armony, J. L., Driver, J., \& Dolan, R. J. (2001). Effects of attention and emotion on face processing in the human brain: An event-related fMRI study. Neuron, 30(3), 829-841. doi: 10.1016/ S0896-6273(01)00328-2

Wieser, M. J., Pauli, P., Alpers, G. W., \& Mühlberger, A. (2009). Is eye to eye contact really threatening and avoided in social anxiety? An eyetracking and psychophysiology study. Journal of Anxiety Disorders, 23(1), 93-103. doi: 10.1016/j.janxdis.2008.04.004

Workman, L., Peters, S., \& Taylor, S. (2000). Lateralization of perceptual processing of pro- and anti-social emotions displayed in chimeric faces Laterality, 5(3), 237-249. doi: 10.1080/713754378

Yarbus, A. L. (1967). Eye movements and visions, New York: Plenum Press.

Received April 22, 2010

Revision received October 27, 2010 Accepted November 15, 2010 